\title{
Development Plan for the Fuel Cycle Simulator
}

\author{
Brent Dixon
}

September 2011

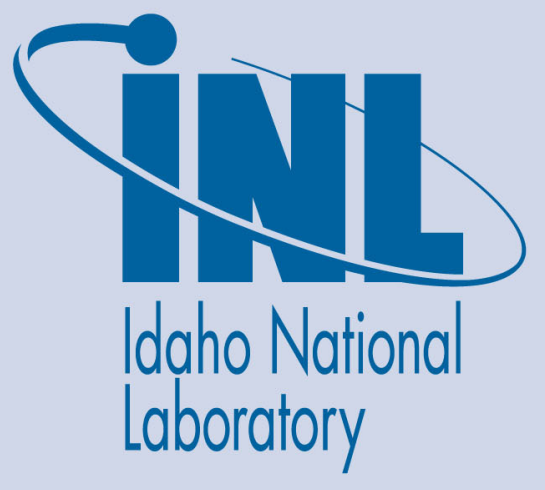

The INL is a U.S. Department of Energy National Laboratory operated by Battelle Energy Alliance 
INL/EXT-12-24541

FCRD-SYSA-2011-000330

\title{
Development Plan for the Fuel Cycle Simulator
}

\author{
Brent Dixon
}

September 2011

\section{Idaho National Laboratory \\ Fuel Cycle Research \& Development Idaho Falls, Idaho 83415}

http://www.inl.gov

Prepared for the

U.S. Department of Energy

Office of Nuclear Energy

Under DOE Idaho Operations Office

Contract DE-AC07-05ID14517 


\section{DISCLAIMER}

This information was prepared as an account of work sponsored by an agency of the U.S. Government. Neither the U.S. Government nor any agency thereof, nor any of their employees, makes any warranty, expressed or implied, or assumes any legal liability or responsibility for the accuracy, completeness, or usefulness, of any information, apparatus, product, or process disclosed, or represents that its use would not infringe privately owned rights. References herein to any specific commercial product, process, or service by trade name, trade mark, manufacturer, or otherwise, does not necessarily constitute or imply its endorsement, recommendation, or favoring by the U.S. Government or any agency thereof. The views and opinions of authors expressed herein do not necessarily state or reflect those of the U.S. Government or any agency thereof. 


\section{CONTENTS}

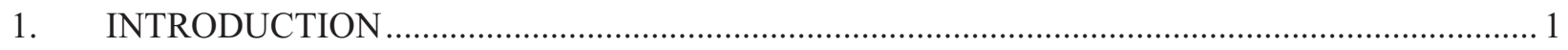

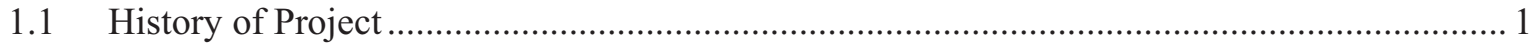

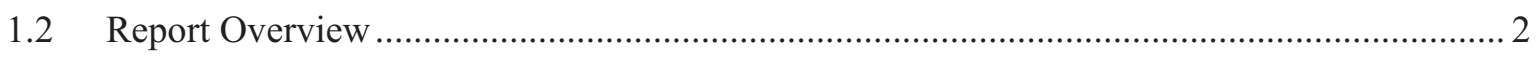

2. ADVANCED FUEL CYCLE SIMULATOR VISION AND OBJECTIVES ................................. 3

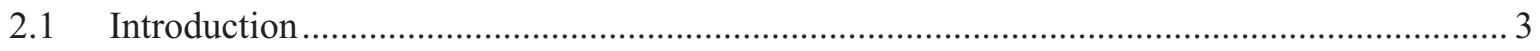

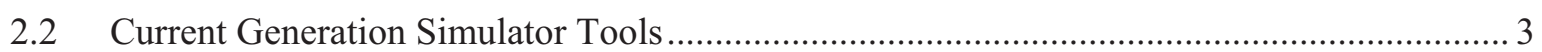

2.2.1 Current Systems Analysis Tools .............................................................. 4

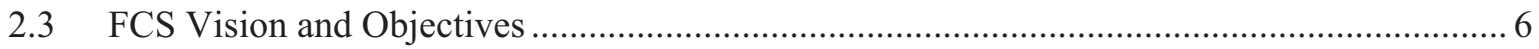

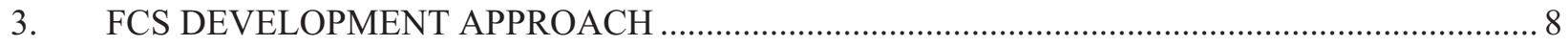

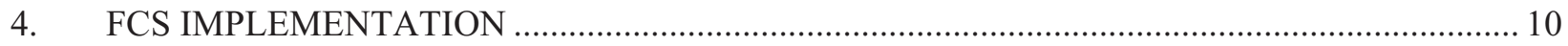

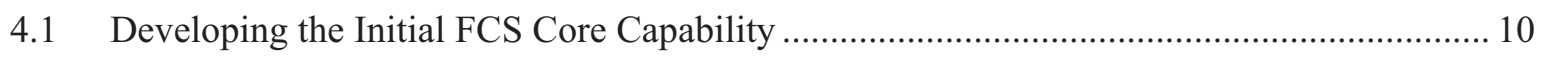

4.2 Establishing the Development Community …............................................................... 10

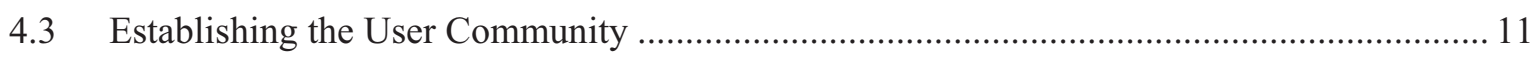

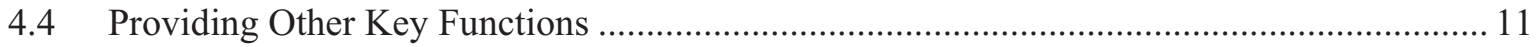

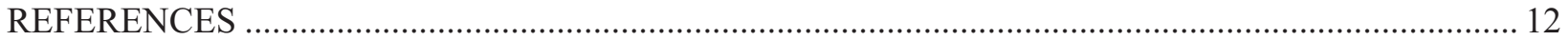




\section{SYSTEMS ANALYSIS CAMPAIGN DEVELOPMENT PLAN FOR THE FUEL CYCLE SIMULATOR}

\section{INTRODUCTION}

The Fuel Cycle Simulator (FCS) project was initiated late in FY-10 as the activity to develop a next generation fuel cycle dynamic analysis tool for achieving the Systems Analysis Campaign "Grand Challenge." This challenge, as documented in the Campaign Implementation Plan, is to:

"Develop a fuel cycle simulator as part of a suite of tools to support decision-making, communication, and education, that synthesizes and visually explains the multiple attributes of potential fuel cycles."

\subsection{History of Project}

The Systems Analysis Grand Challenge was initially identified a couple years ago. In FY-10, initial functions and use cases for the FCS were developed, along with a vision for long-term FCS development as an open source project [1]. The concept drew significant interest from multiple parties as a way to provide an integrated analysis capability similar to the Yucca Mountain Project's Total Systems Model, while also assisting in improving communication of fuel cycle issues to non-technical stakeholders via advanced visualization techniques. In the summer of 2010 DOE decided to proceed with development of the system.

The original concept for developing the FCS was via a Nuclear Energy University Program (NEUP) "Integrated Research Project" (IRP), a new grant vehicle where a single team of universities is given full responsibility for planning, developing, and delivering an integrated product based on resources of $\sim \$ 2.5 \mathrm{M}$ per year over a three year period. The IRP is in contrast to the primary NEUP program, where several smaller, independent research projects are funded in each area of nuclear R\&D. Lab funding would be sufficient to monitor the IRP effort. The FCS was one of three IRPs proposed for FY-11.

The budget pass back from the Office of Management and Budget only authorized two IRPs for FY-11, so the FCS development approach was reconfigured. The new implementation strategy for the effort involved a ramp-up of laboratory funding to support construction of core capabilities, and funding of several regular NEUP grants to support development of additional capability modules that could be linked to the core program. These modules would include both analytical models of portions of the fuel cycle and modules for user interfaces and visualization of analysis results. The total effort would span 3-4 years with funding levels comparable to what the IRP would have provided.

A baseline change request was processed to set up $\$ 700 \mathrm{~K}$ of FY-11 laboratory activities via carryover reallocation, while a supplemental NEUP call was issued for NEUP proposals. Laboratory scope included a series of workshops as input to a full requirements development activity and initiating an advisory group for the project. A well-attended workshop was held in May, 2011, resulting in significant information for the requirements effort. 18 NEUP pre-proposals were received for the FCS call, with 8 encouraged to develop full proposals. The full proposals were received and ranked, with an initial funding target of $\$ 2.5 \mathrm{M}$ (over 3 years). The approach included funding additional NEUPs in subsequent years, such that total funding would be similar to what would have been available via the IRP.

In June, DOE decided to significantly scale back the FCS effort, reducing the NEUP portion to a single $\$ 1.2 \mathrm{M}$ three year grant and the laboratory portion to monitoring of that grant. Plans for additional workshops, a requirements document, and the advisory board were cancelled. The revised scope was 
limited to modifying the NEUP grant to be a standalone effort and writing a new development plan based on the new level of resourcing.

Given funding of a single NEUP project involving four universities along with only minimal laboratory funding, the updated development approach is to follow a scaled down version of the IRP. Over the next three years at a funding level of $\$ 400 \mathrm{~K} / \mathrm{yr}$, the university team will develop and demonstrate the basic capabilities of FCS using a modular architecture that will provide a platform for further evolution.

\subsection{Report Overview}

This report documents the current FCS development plan. Chapter 2 describes the vision behind FCS development and discusses the objectives for the FCS. Chapter 3 provides an overview of the architecture for the FCS simulator. Chapter 4 discusses the overall approach for the project, including both the university and laboratory efforts. This includes how the handoff of the university product is expected to proceed and what comes after the NEUP is completed.

The development plan is intended to provide the general direction for the effort without being overly prescriptive. The project has already gone through 2 significant changes, and more changes may occur. One such change will be if additional FCS-related NEUP grants are funded in future years. The current grant involves four universities and 5 co-Principle Investigators. A second potential change will be if a specific role is identified for the FCS in planning fuel cycle option screening activities, which would potentially provide additional resources while likely invoking new schedule constraints. 


\section{ADVANCED FUEL CYCLE SIMULATOR VISION AND OBJECTIVES}

\subsection{Introduction}

A fuel cycle simulator is an analysis tool used to understand the operation and evolution of a complete nuclear energy system. The nuclear fuel cycle starts with the mining of uranium or thorium ore, proceeds through the fabrication and irradiation of nuclear fuel, and ends with the disposal of nuclear waste. A simulator dynamically models the flow of materials and the operation of the infrastructure that stores, transports, and converts those materials. Fuel cycle options include linear "once-through" systems and closed-loop "full recycle" systems, often in combination.

Dynamic simulation models are used to examine systems in transition, such as occurs when a fuel cycle strategy changes to include recycling. Analysis scenarios include assumptions on changes in total energy generation with time, as well as when new technologies become available or when strategy changes are to be initiated. Simulation timeframes are typically 100 years or more.

Analyses provide information on sufficiency of materials for startup of new reactors, capacities and timing of fuel cycle facilities needed to support the growth and/or transition of the total nuclear energy system, and characteristics and quantities of materials throughout the system. The material characteristics and quantities are used to understand changes in material attractiveness (for proliferation risk and security comparisons) and waste parameters (waste loading, decay heat, source terms, etc.).

Appropriate uses of analysis results include identifying changes in behavior (inflection points on time plots of parameters) and understanding their drivers and their consequences. The exact timing and magnitude of the changes are usually less important than the reason behind the trend. When such a trend is identified, sensitivity analyses of input parameters help to better understand the phenomenon.

\subsection{Current Generation Simulator Tools}

Several governments, companies, universities, and international organizations have developed simulators of fuel cycles and nuclear energy systems. One of the first steps in the development of the FCS project was to assess several of these simulators that were in current use or active development for both common and unique characteristics and limitations [1]. This effort borrowed heavily from another recent simulation scoping study for the SINEMA program [2], [3]. The FCS project seeks to employ lessons learned with current fuel cycle simulation tools while also understanding how the underlying architecture has shaped their development.

Through the review it was confirmed that existing codes have a range of capabilities that are desirable for the FCS to incorporate (e.g. multi-regional analysis, detailed isotopic tracking, cost analysis, etc.). However, while all of these codes are capable of supporting at least a subset of the range of fuel cycle analyses which are performed within government, universities, and industry, none supports the superset of these analyses. An individual code typically performs detailed analysis of a few particular fuel cycle areas, reporting detailed indicators for those aspects while neglecting others. For example, some codes that focus on economics neglect detailed isotopic tracking, while others carefully track and calculate waste disposal metrics while neglecting cost-analysis and multi-regional interactions. Furthermore, while it may seem that linking these codes to each other could provide a tool with an aggregate set of their capabilities, a self-consistent tool would not result from such a linkage.

Part of the vision for FCS is to develop a tool that can meet everyone's needs; the superset of current capabilities as well as new capabilities. 
Another observation from the review is the trade-off between accuracy and functionality. Depending on the types of analyses to be performed, more accurate modeling of the real world may result in revealing additional behavior modifiers or may just add random variations to the simulation results that inhibits identification of trend changes. An example is the explicit modeling of individual fuel reloading batches versus just modeling the average annual fuel flow. Figure 1 is from the final report of the MIT-led benchmarking of four simulation codes [5]. For some applications, the additional information on individual reloads provided by the French code COSI is very useful, but for general communication, these details are distracting.

\section{One lesson learned is the need to have flexibility in both how analyses are conducted and how results are presented.}

Several specific capabilities were identified that are desired for the FCS, including discrete modeling of facilities and materials, modeling of unconventional reactors, the ability to assess system disruptions, modeling of uncertainty and automation of sensitivity assessments, open access to source code for model extension, and incorporation of dynamic feedback of economics as part of the logic that guides scenario development. Some of these features were at least partially implemented in a few existing codes, while others were identified as gaps that prevented current codes from being used to analyze certain families of problems. The draft FCS functions and requirements report includes an extensive list of suggested functions and "use cases" (specific types of analyses).

On the interface side, the generic desired capability is to be able to "zoom in" to investigate specific behavior and understand both its cause and consequences. Referring to Figure 1, it is not sufficient to notice that uranium usage briefly increases before declining as fast reactors are introduced. The user needs to be able to expand the timeframe around that transition, and then pull up other aligned graphs showing what related parameters are doing. Information of dependent and independent parameters will help the user to identify the cause. Consequences could be highlighted by "rocking" the simulation (running it backward and forward) while modifying the input parameters. For the non-technical user, this all needs to be accomplished while describing the affected parameters in "plain English".

\subsubsection{Current Systems Analysis Tools}

The review also identified the value of developing FCS initially as a supplement to the current suite of nuclear systems analysis tools used by the DOE Fuel Cycle Technologies (FCT) program. This specifically includes being a complement to VISION, the current fuel cycle simulation code [4], and not a direct replacement. One of the primary drivers for this position is the differences is the modeling architecture; systems dynamics for VISION versus object/agent for FCS.

The VISION systems dynamics architecture is ideal for modeling multiple interacting attributes of a fuel cycle but is limiting in its ability to model multiple instances of specific attributes such as facilities or fuel batches. Due to the long operating lives of reactors and fuel cycle facilities, fuel cycle evolutions occur over periods of a half century or more. Assumptions about individual facilities become more generic at longer time periods, so a fleet average approach provides a reasonable level of accuracy given the input assumptions without modeling excessive details. 


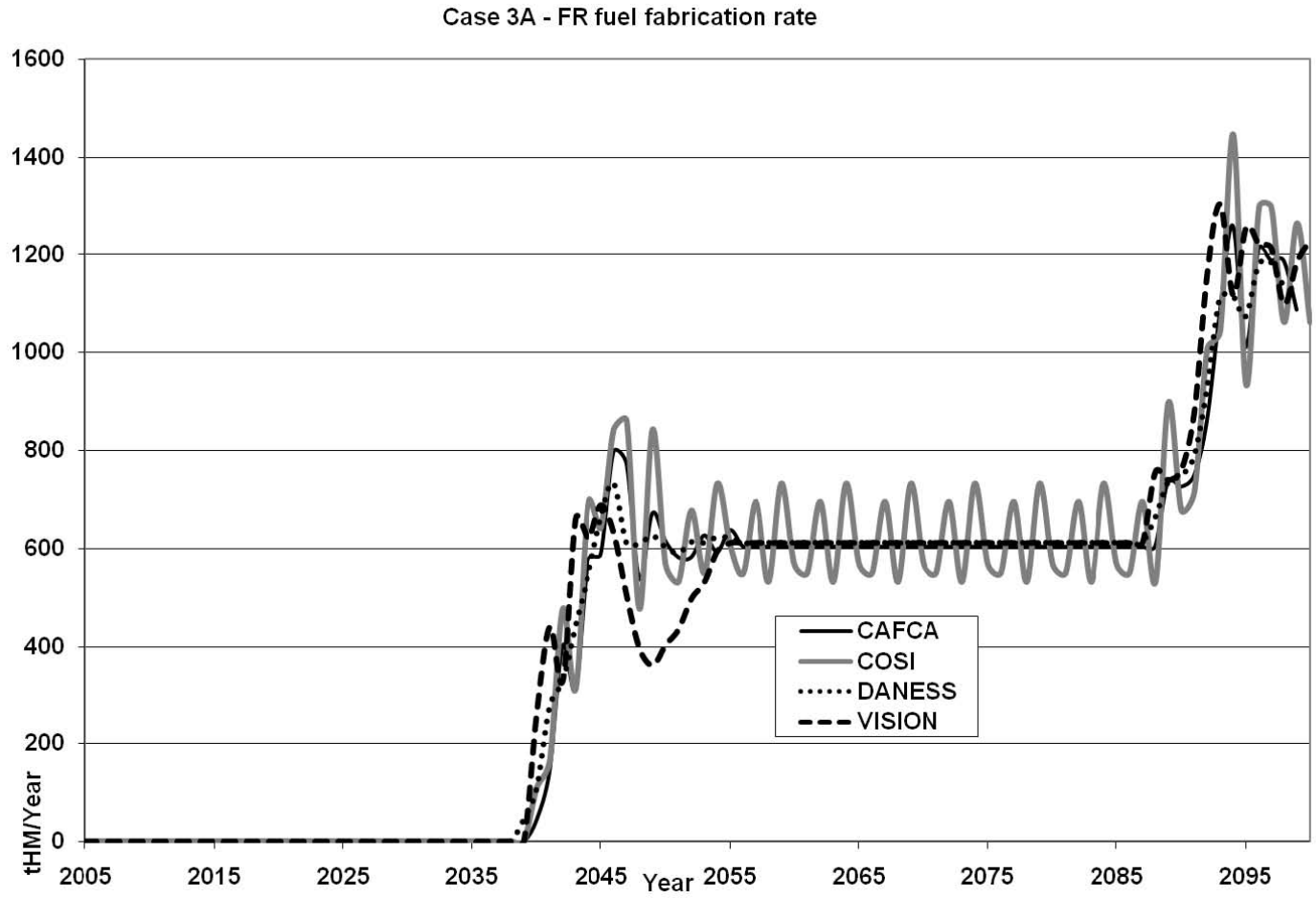

Case $3 A$ - Nat. U consumption rate

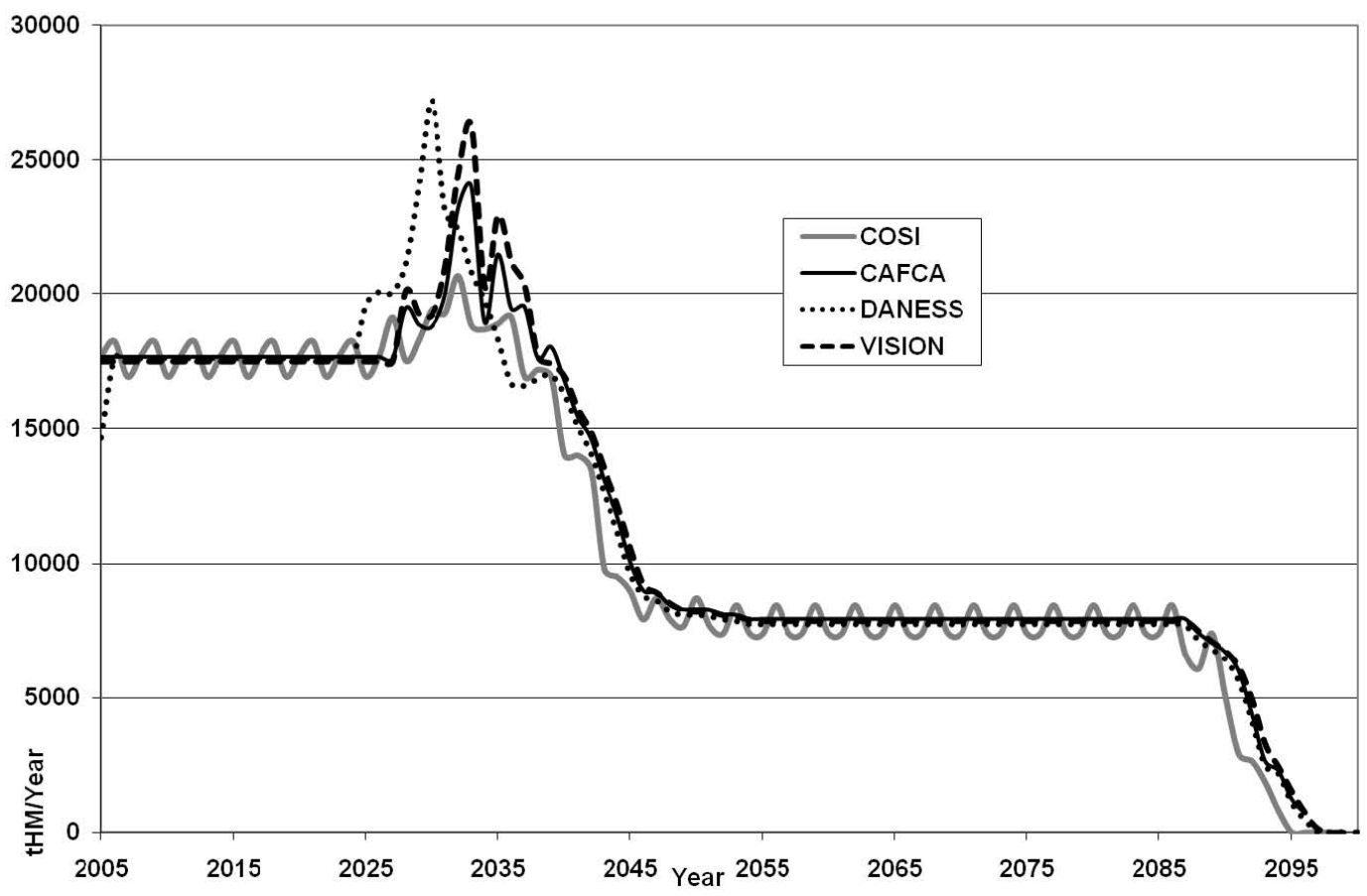

Figure 1 - Benchmarking example of fuel cycle simulation codes showing the impact of fast reactor introduction 
VISION is targeted for analysis problems involving fleet average behavior and total fleet capacity evolution, but not the behavior or differentiation of individual facilities - VISION is limited to 10 total reactor types, where there can be multiple reactors within each type but most of the modeled behavior is for the reactor type as a group. The exception is that new builds and retirements are modeled by time step $\left(1 / 4^{\text {th }}\right.$ year), but the general treatment is still a total capacity installed or retired of that reactor type during that time step. The same is true for fuel cycle facilities, but since there may be only limited numbers of these facilities in a particular scenario (e.g. only one reprocessing plant) then there is the appearance of modeling each facility individually.

VISION is based on a systems dynamics architecture that, like many of the existing codes, includes modeling fleet average behavior but not individual reactors. This makes VISION very useful for certain classes of problems where the added detail of modeling discrete facilities is not needed. While the fleetlevel modeling in VISION is ideal for assessment of general behavior and trends, it does not support other types of fuel cycle analyses. The FCS object/agent architecture will support modeling of individual reactors and fuel cycle facilities, along with several other types of entities not supported in VISION.

Initially, FCS will be positioned to complement existing tools by providing capabilities they don't have. Later, FCS may evolve to the point that many existing tools can be retired.

The original design parameters for VISION included operation on a standalone desktop computer with a runtime of 5 minutes or less. The full VISION implementation, including the code, input/output files, and libraries has grown over time to roughly $200 \mathrm{Mb}$. As more capabilities have been added, the run time goal has only been achieved by updating to the latest (fastest) personal computers available.

FCS will be developed to operate in a virtual hardware environment (cloud computing), where a local device will provide the user interface, while other hardware provides the computational and data storage capabilities needed to achieve performance goals.

The Systems Analysis toolbox includes other tools at varying levels of maturity. The Fuel Cycle Integration and Tradeoffs model (FIT) [6] provides static analysis capabilities which can address the impacts of material processing losses and isotopic composition differences at a more detailed level than VISION. FIT is written in Excel. Two Matlab-based tools are also currently under development, including one for analyzing uranium supply/demand and one for cost modeling of fuel cycle options. Finally, funding was recently allocated for development of a facility level simulation of an aqueous separations plant. As each tool matures, a decision will be made to either maintain it separately or merge it with FCS.

A modular architecture will improve the potential to merge existing tools into the FCS.

\subsection{FCS Vision and Objectives}

The vision for the FCS is a joint effort by DOE laboratories, universities, industry and potentially international partners to combine intellectual resources into a single, next generation software tool that can meet the needs of all users.

- Use the best features and lessons learned from current generation tools, their developers, and their users (and potential new user classes) to develop the functional requirements

- Apply advanced software development and visualization methods and advanced hardware architectures to achieve a maintainable and extendable tool that satisfies those requirements 
Objective 1 - Significantly improve on the human/computer interface of the simulator.

Current simulators are tools used by experts with code results often only understandable by experts. The FCS vision includes also using the simulator to inform and educate non-experts, including policy developers, students, and other stakeholders.

The intent is to extend the types of users while providing these users with a completely different way to interact with the computer to investigate and communicate important findings of analyses. Proven advanced visualization techniques will be investigated to extend access of the FCS to this broader base of users and viewers.

The interface will also need to support flexible access to both real-time and archived analyses. Provenance features need to be included in the database to enable seamless restart of archived scenarios.

Objective 2 - Establish extensive communities of developers and users.

Today, every organization builds their own simulator. Through collaboration, we hope to eventually eliminate the current duplication of effort in development and maintenance.

An international colleague indicated that for most of their work, they used a full-featured simulator developed by another country. However, they were also continuing to develop their own tool, because they were not allowed to modify the other tool and it did not support some specific capabilities they lab needed. A modifiable open source code would enable this lab to model the specific interests of their government without the expense of developing and maintaining their own code.

Several recent nuclear engineering dissertation efforts have involved development of limited fuel cycle simulators as research tools. In each case, significant effort was expended to obtain a functioning simulator, with only moderate additional effort to add the special analysis features supporting the research. If instead these efforts started from an existing code platform, the software portion of the effort could have been minimized, enabling a more in-depth investigation of the nuclear issues.

A modified open source approach is being pursued for FCS, modeled after other successful efforts. This will enable a distributed FCS development environment supporting participation from labs, universities and industry, domestic or foreign. A modular architecture will allow developers to build upon and extend the work of others, eventually providing much more functionality than existing codes.

Once the initial FCS functionality is in place, we hope to establish a large community of users. This will stimulate additional development while justifying the hotel costs for maintaining the effort.

Objective 3 - Use current distributed computing technologies to provide a flexible, extendable, and maintainable platform for the FCS.

The advanced interface and expanded functionality of the FCS will require additional computational power, while users desire delivery on laptops and tablet computers. The computer science community has identified architectures that will support these requirements while also supporting distributed development and selective reuse of legacy code. These public domain solutions will truly make the FCS a next generation tool, using the best of current generation equations, algorithms and data while supporting growth of functionality well beyond the ability of all current systems. The use of public domain solutions will minimize or eliminate the need for commercial software licenses - a significant expense for VISION that inhibits university use. 


\section{FCS DEVELOPMENT APPROACH}

As stated previously, the DOE FCT program has an existing fleet-level simulator, as do the national nuclear fuel cycle research programs of several other countries. These simulators are typically complex, tracking mass at the isotopic level, and are focused on the areas of concern for the sponsoring programs. They primarily focus on the back-end of the fuel cycle and support analyses of advanced fuel cycles with different levels of recycling. Universities also have fuel cycle simulators of varying complexity, usually focused on research for advanced fuel cycles or advanced reactors, and usually developed and extended by graduate students under faculty leadership. The fuel cycle simulation of industry is quite different, typically focusing on the front end of the current once-through uranium-based fuel cycle, assessing economic decisions related to commercial operation.

The approach for the FCS is to use an architecture and software development approach that will support additional classes of analyses while improving flexibility and extensibility, reducing software development and maintenance costs, and encompassing the needs of government and university research, education and communication, while being extendable to also support industry applications.

The first step in development will be to establish the core capabilities necessary to have a working simulator in the target architecture. This will be achieved over the next three years by a team of four universities, led by the University of Wisconsin. A modular object/agent based software approach will be implemented in a distributed development and operating environment. This approach will enable the following key capabilities:

- Analysis of new classes of problems - For example, the new architecture will allow full cost modeling of individual future facilities owned by regulated and unregulated utilities, enabling assessment of different government incentive approaches to encourage industry adoption of advanced technologies and fuel cycles.

- Flexible evolution of simulation capabilities - For example, exchangeable modules will incorporate different approaches to the modeling of transmutation behavior to allow for rapid execution using pre-calculated isotopic "recipes" when accuracy is less important versus performing on-the-fly neutronics calculations when high accuracy is needed. The same capability will allow for modeling of other fuel cycle facilities, such as a fuel fabrication plant, as anything from a simple transformation matrix to a detailed model that includes process losses and associated wastes from each fabrication stage.

- Participation by multiple organizations in FCS development and use - The modular approach will allow a PhD candidate to develop a code extension assessing the impact of different heterogeneous core approaches on minor actinide management as part of her dissertation research, without having to also build the balance of the fuel cycle. It will also enable linking a detailed DOE laboratory simulation of a separations plant to the rest of the fuel cycle, to examine the impact of upstream feedstock variations and downstream fuel fabrication compositional requirements on the plant's operations and design.

- Support for a wide range of users - Advanced visualization tools coupled with simplified modules and default parameter values will enable users such as undergraduate students and policy staff to develop a basic understanding of how a fuel cycle works and how performance is constrained. The same advanced visualization tools will also enable expert users to identify new performance nuances and explore their causes. 
The core capabilities will include:

- Computational modules for each stage of the fuel cycle. These modules will be simplistic in the initial implementation, as the goal is to have a system working within the new architecture. Any future developer will be able to extend these modules to provide more sophisticated capabilities. When feasible, equations used in current generation simulators will be documented and reused in the FCS effort.

- A client-server or cloud computing virtual architecture that will allow any networked computer to serve as a user interface to the system. This architecture is still to be developed, but the concept is to use public domain software so that no licenses are required of users.

Figure 2 shows the concept for this environment. The user interface will run on the user's computer. The other applications will be able to run on other computers, assigned dynamically without user intervention. In the initial application, this may be just a simple client-server system with one server computer.

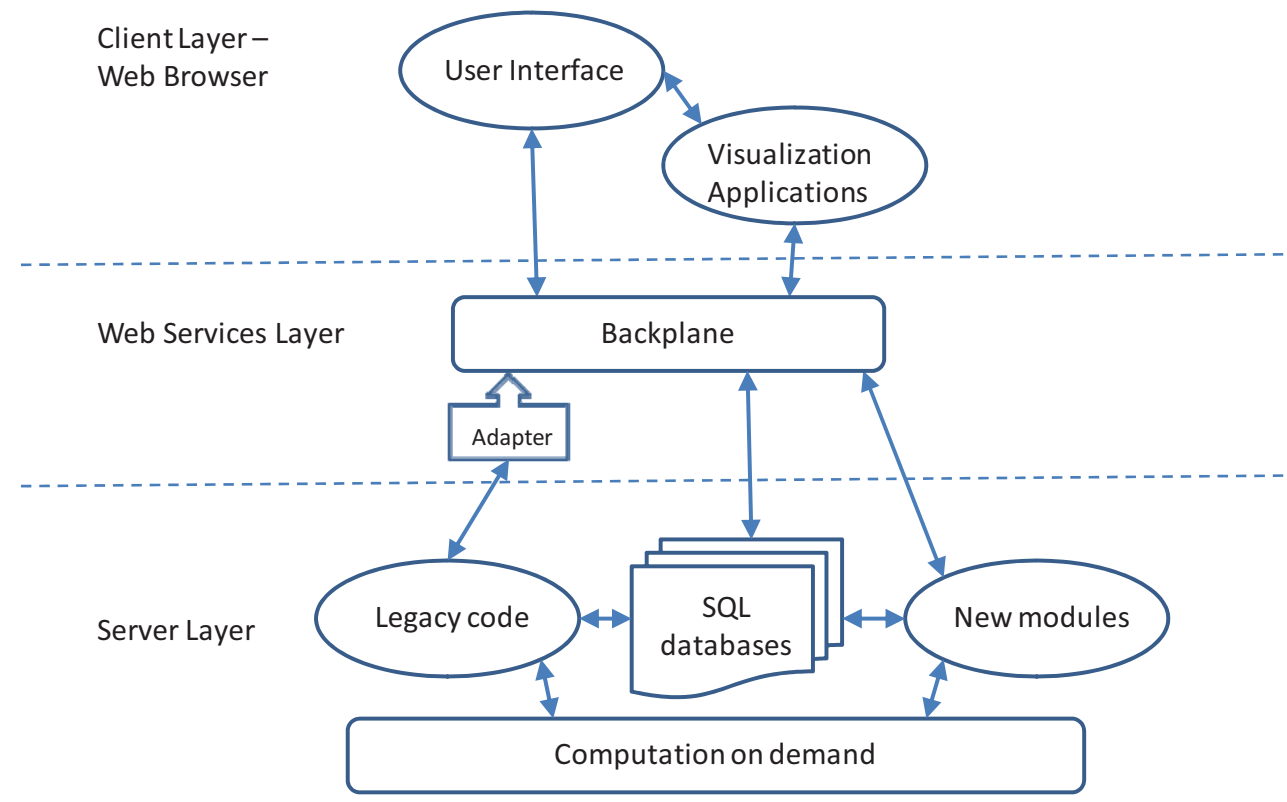

Figure 2 - FCS Virtual Environment Concept

- A database or set of databases including libraries of transmutation data, cost data, etc., along with input parameter sets for different scenarios and storage of analysis results.

- A user interface for building and running simulation scenarios. A drag-and-drop approach is envisioned for the initial interface.

- A visualization environment for analytical reasoning and data exploration. This environment will be linked to the simulation module and database, enabling different methods of interactively displaying analysis information. This is an important research area for the initial FCS project, as a number of visualization applications have been developed for other data domains and their utility for FCS needs to be determined. This specifically includes working with potential nontechnical users to determine how best to support their information and exploration needs.

- If appropriate, some computation modules may be implemented by using existing code. These legacy applications will likely require an adapter layer to interface with the rest of the system. 


\section{FCS IMPLEMENTATION}

Several actions will be needed to fully achieve the FCS vision of a shared development effort producing a single, next generation software tool that can meet the needs of all users.

\subsection{Developing the Initial FCS Core Capability}

The funded NEUP project will provide the initial core system implemented in the target architecture. This system will have limited capabilities, especially when compared to the list of desired use cases in the draft functions and requirements document [1]. To be successful, it must only provide a catalyst capability sufficient to attract additional development.

The key to success of the overall FCS effort will be establishing a community of developers and users beyond the initial core effort. The vision requires FCS to become the standard fuel cycle analysis platform, not just for the U.S. national laboratory system, but also for universities, policy think tanks, industry, international activities, etc.

\subsection{Establishing the Development Community}

Given the current resource limitations, the true grand challenge may end up being the effort to establish a critical mass of developers to extend FCS capabilities beyond the initial core. The 3-year duration of the current NEUP grant provides significant time to develop other support. A multi-pronged approach will be used:

- Encourage the university community to participate in extending FCS rather than initiating new standalone efforts. Every year, nuclear engineering graduate students develop new software to support their research. If the universities see this as a group effort, and not just the domain of the core team, then they could join in via graduate student-developed code contributions.

- Seek additional NEUP opportunities for participation. This could include new grants to complement the initial one, funding additional universities to participate in the development effort. It could also include encouraging recipients of NEUP fellowships to do FCS-related research.

- Explore interest by other (non-nuclear) university departments. For example, FCS can provide an opportunity for research in the social sciences on how to communicate technical matters to nontechnical stakeholders that could result in enhancements to the FCS visualization modules.

- Explore interest by the international community. Several recent efforts have assessed the capabilities of current codes and found room for improvement to address new types of problems. In addition, several countries do not have fuel cycle codes and are not satisfied with the code suite offered by the International Atomic Energy Agency. Canada and Korea have both expressed interest in using the VISION code, and an analyst from the Korea Atomic Energy Research Institute is traveling to the INL this month for VISION training. An international collaborative effort could provide an opportunity for countries with smaller research efforts to share ownership and control of a full featured code as an alternative to developing their own codes with more limited capabilities.

- Explore interest by U.S. industry. EPRI was set to sponsor the next FCS workshop before it was cancelled. The Nuclear Waste Technical Review Board has been developing their own code and recently hosted a benchmarking workshop. If these organizations were interested in participating in a joint effort, they may be able to attract others. Feedback on an FCS collaboration could also be solicited from the six nuclear industry groups currently under contract with DOE. 
- Tie in DOE lab developed tools, such as the recently funded separation facility simulation. While DOE has cut direct funding for FCS in FY-12, development of other tools continues. New direct funding for FCS is also possible in future years, but may be best allocated to providing other key features.

\subsection{Establishing the User Community}

The strongest driver for development is to have broad use of the product. A vibrant FCS user community will help justify further development while also providing direction for that development. Some users may also become developers themselves.

The first FCS users will be members of the initial core development effort. To grow beyond that group the capabilities generated by the NEUP grant must be sufficiently attractive. The FCS is planned to provide new analysis functions not available on other simulators. This will be the initial basis for attracting additional users.

The sources for FCS users are the same as for FCS developers, so the same areas will be worked to generate user interest as were listed in the previous section. In addition, the following actions can be pursued:

1. Actively work to expand VISION users while the FCS core is under development. VISION was originally developed to model only the U.S. commercial industry, and then only for a transition to fast burner reactors. The original program was also limited to use by U.S. citizens. In the last year VISION was approved for export and VISION capabilities were expanded to be able to model any generic fuel cycle. Both of these developments make VISION more attractive to universities and other users. An active VISION user community can be a source of FCS users.

2. Establish a formal users group for FCS. By setting up a users group up front, initial users will have input into development and will develop a degree of ownership. Assuming the initial FCS is a sufficiently useful product, the initial users will promote it with others. As the FCS evolves with additional user requested capabilities, this will have a snowball effect.

\subsection{Providing Other Key Functions}

Along with developers and users, some other key functions are required to sustain a successful program. First, there must be a set of standards for programming and documentation. The initial standards should be established (and followed) by the NEUP development team.

After the NEUP development team turns over their product, a maintenance and V\&V group will need to be established. This group needs to be independent of the developers. They will enforce the standards, provide version control of FCS, check and test new modules offered by developers before including them in official releases, keep libraries up to date, and manage bug fixes. This must be an ongoing, funded effort, though the level of effort is TBD. Initially FCS maintenance could probably be included with the current maintenance of other Systems Analysis tools.

Establishment of an advisory committee was included in the original FY-11 FCS work scope. The committee was intended to initially provide independent advice on FCS architecture choices and other technology matters, as well as recommendations on the overall development direction of the FCS. However, following the model of a "mini IRP", the architecture, standards, and other initial technology matters are the responsibility and authority of the NEUP team developing the core capabilities. This committee is still recommended, but unless significant additional developers appear in the near term, the committee does not need to be established until the NEUP effort is nearing completion. 


\section{REFERENCES}

[1] K. Huff and B. Dixon, "DRAFT Next Generation Fuel Cycle Simulator Functions and Requirements Document," INL/MIS-10-19679, Idaho National Laboratory, July 2010.

[2] C A Juchau, M L Dunzik-Gougar, and J J Jacobson, Modeling the Nuclear Fuel Cycle. Nuclear Technology. Vol. 171 (2010), 136-141.

[3] A Miron, J Valentine, J Christenson, M Hawwari, S Bhatt, ML Dunzik-Gougar, M Lineberry. Identification and Analysis of Critical Gaps in Nuclear Fuel Cycle Codes Required by the SINEMA Program. DOE/ID/14839. (2009).

[4] A M Yacout, J J Jacobson, G E Matthern, S J Piet, D E Shropshire, C T Laws, "VISION - Verifiable Fuel Cycle Simulation of Nuclear Fuel Cycle Dynamics," Waste Management 2006, February 26March 2, 2006, Tucson, Arizona.

[5] "A Benchmark Study of Computer Codes for System Analysis of the Nuclear Fuel Cycle," Nuclear Fuel Cycle Technology and Policy Program, Massachusetts Institute of Technology, MIT-NFC-TR105, April 2009.

[6] S. Piet, N. Soelberg, L. Pincock, E.Shaber, and G. Teske, "The FIT 2.0 Model - Fuel-cycle Integration and Tradeoffs," INL/EXT-10-20190 rev 1, Idaho National Laboratory, June 2011. 